\title{
Aspectos regulatorios del teletrabajo en el Perú: análisis y perspectivas*
}

Regulatory Aspects of Teleworking in Peru: Analysis and Perspectives

\section{Ana Maria Cecilia Valencia Catunta“*}

\begin{abstract}
RESUMEN
La presente investigación busca dar a conocer el tratamiento del Teletrabajo en el Perú, modalidad de prestación de servicios para losámbitos público y privado, en la que se aplica las Tecnologias de la Información y las Comunicaciones (TICS), constituyendo su regulación un aporte para la ciudadania, particularmente para quienes no pueden desplazarse físicamente a un centro de trabajo, y para el desarrollo de los teletrabajadores. Asimismo, el Teletrabajo se vincula con la política pública de Gobierno Electrónico, transversal a todas las políticas estatales y constituye una herramienta que promueve la protección de los derechos humanos y el desarrollo del pais al generar empleo, optimizando la productividad y mejorando la calidad de vida de los ciudadanos.
\end{abstract}

\section{PALABRAS CLAVE}

Teletrabajo, tecnologias de la información y las comunicaciones, Gobierno electrónico, política pública, calidad de vida.

\begin{abstract}
The present research seeks to show the treatment of Telework in Peru, a form of provision of services for the public and private sectors, in which Information and Communication Technologies (ICTs) are applied, and its regulation constitutes a contribution to the citizenship, particularly for those who cannot physically move to a workplace, and for the development of teleworkers. Likewise, Telework is linked to the public policy of Electronic Government, transversal to all the state policies and constitutes a tool that promotes the protection of human rights and the development of the country by generating employment, optimizing productivity and improving the quality of life of their citizens.
\end{abstract}

\section{KEYWORDS}

Telecommuting, Information and Communication

Technologies-ICT, Electronic Government, Public Policy,

Quality of Life

*Artículo recibido el 9 de junio de 2017 y aceptado el 23 de agosto de 2017

**Universidad Tecnólogica del Peru-Utp, Universidad Norbert Wiener (avalenci2002@yahoo.es) orcid 0000-0001- 
SUMARIO

1. Introducción

2. Antecedentes

3. Definición del teletrabajo

4. Características del teletrabajo

5. Beneficios y calidad de vida

6. Aspectos resaltantes de la Ley 30036 y su Reglamento

7. Políticas públicas y el teletrabajo

8. Teletrabajo y gobierno electrónico

9. Conclusiones

\section{Introducción}

Las tecnologías de información y las comunicaciones (тіс) constituyen un medio indispensable para el desarrollo de los países. Tal es su relevancia que se ha intensificado su aplicación en los ámbitos privado y público. Así, en el Perú, por ejemplo, se ha regulado como política pública el desarrollo y aplicación de las TIC, la cual es transversal a todas las políticas públicas que se formulan para la solución de los problemas de diversa índole que aquejan a los ciudadanos, particularmente a las poblaciones vulnerables. Uno de ellos es el acceso al trabajo necesario para satisfacer otros derechos humanos y para gozar una vida digna. Por ello, corresponde a los Estados, que tienen la obligación de respetar, proteger y garantizar el derecho al trabajo, generar oportunidades laborales sin discriminación y a las cuales se pueda acceder libremente.

El teletrabajo viene aplicándose exitosamente en diversos países, dadas sus ventajas para el teletrabajador, así como para la empresa y la sociedad. Por tanto, ha sido regulado en el Perú. Lo promueve el Ministerio de Trabajo y Promoción del Empleo por ser el trabajo, en sus diversas modalidades, atención prioritaria del Estado peruano conforme a su Constitución Política.

El teletrabajo es una modalidad especial de prestación de servicios donde se utilizan medios informáticos y de telecomunicaciones. Esto lo diferencia de otras figuras que se le asemeja. Permite que los ciudadanos, especialmente aquellos que conforman poblaciones vulnerables, y las empresas tengan la oportunidad de mejorar su calidad de vida y productividad, respectivamente.

Así también, el teletrabajo constituye una opción laboral que promueve y brinda protección a los derechos fundamentales (vida, salud, educación, entre 
otros), ya que permite crear fuentes de trabajo. Por ello, los Estados deben garantizar su desarrollo y uso. Sobre lo señalado, el Estado peruano reconoce las ventajas del teletrabajo y expresamente regula la necesidad de garantizar su desarrollo a través de políticas públicas.

En relación a lo expuesto, es nuestro interés dar a conocer los aspectos más relevantes del teletrabajo en el Perú: las entidades y empresas que contratan teletrabajadores, las expectativas que se tiene con su promoción, así como las políticas públicas para su aplicación y la entidad del Estado responsable de su desarrollo.

\section{Antecedentes}

El teletrabajo surgió en el año 1973. El padre de dicha modalidad laboral fue el físico Jack Nilles. Con el apoyo de un grupo de científicos de la Universidad de California del Sur, estudió el uso de tecnologías informáticas para resolver el problema que afrontaban las empresas por la crisis del petróleo. Se llegó a establecer que el empleo de las Tic no sólo beneficiaba a las empresas en sus costos, sino que protegía el medio ambiente por la disminución en la emisión de gases nocivos. Los estudios de Jack Nilles, asimismo, beneficiaron tanto al ámbito privado como al público. Se formularon políticas públicas para su utilización en la organización del trabajo. Las empresas multinacionales norteamericanas y luego Europa utilizaron el teletrabajo y consideran sus bondades, pese a que los problemas que originaron su nacimiento continúan sumado a ello la competencia empresarial por la globalización económica. ${ }^{1}$

Vargas señala:

Una de las primeras referencias del teletrabajo la tuvo Alvin Toffler, en su obra La tercera ola (la primera edición de su libro fue en el año 1980), al predecir la aparición de una nueva modalidad de trabajo en el hogar basada en las nuevas tecnologías, en donde un "[n]uevo modo de producción hace posible: un retorno a la industria hogareña sobre una nueva base electrónica y con un nuevo énfasis en el hogar como centro de la sociedad [...].” y que Jeremy Rifkin, "dieciséis años después (1996), confirmó el pronóstico de Toffler al señalar que: “[...]

\footnotetext{
${ }^{1}$ Cfr. Ushakova, TATSIANA, "El Derecho de la oI para el trabajo a distancia: ¿una regulación superada o todavía aplicable?" Revista Internacional y Comparada, pp. 5-6. Disponible en: http://www.europeanrights.eu/public/commenti/ Bronzini21-USHAAKOVA-332-686-1-PB.pdf
} 
en los Estados Unidos entre 1992 y 1993 el número de trabajadores a distancia se estima que creció en un 20\%. Cerca de 8 millones de personas utilizan las telecomunicaciones. En el año 2000, de acuerdo con un estudio realizado al respecto, hasta el $20 \%$ de la masa laboral de los Estados Unidos trabajará, aunque sea parcialmente, desde su propia casa. $^{2}$

Asimismo, a finales de la década de los noventa se tuvo una visión del teletrabajo o trabajo a distancia como una herramienta que beneficia a las partes intervinientes: empleadores y los empleados o teletrabajadores. Además, constituye un instrumento de inserción laboral para personas vulnerables (mujeres y personas con discapacidad física). ${ }^{3}$

La palabra teletrabajo proviene de la raíz griega thele, que significa que algo es lejano o a distancia. Así, en Colombia, con base en un estudio realizado a más de 1740 empresas de todo el país, se observó que los teletrabajadores pasaron de 31553 en 2012 a 95439 en 2016. Las ciudades con más plazas de trabajo en esta modalidad son Bogotá (55 948), Medellín (25 081) y Cali (5723). De este total, 55168 teletrabajadores pertenecen al sector servicios y 27128 al sector de la industria. También aumentó la productividad de los teletrabajadores en 47\%. Con ello mejoró su calidad de vida. Los medios de comunicación más empleados son la telefonía móvil, la mensajería instantánea y la videoconferencia. ${ }^{4}$

\section{Definición del teletrabajo}

Debido al tratamiento que diversas disciplinas le han dado, no es posible encontrar una definición única de teletrabajo. Por ello, daremos a conocer algunas definiciones que se plantean sobre el teletrabajo. Así, el teletrabajo o contrato a distancia "Es la realización de actividades de índole laboral emanadas de un contrato de trabajo, en un lugar distinto a las instalaciones del empleador, que generalmente es el domicilio o residencia del teletrabajador, quien, asistido

\footnotetext{
2 VARGAS RASCHIO, TIno, La respuesta del ordenamiento laboral peruano frente al teletrabajo, Tesis para obtener el título profesional de abogado, San Miguel, 2010, pp. 5-6.

${ }^{3}$ CASTRO, Luis, Propuesta para un modelo de teletrabajo en la Universidad de Costa Rica, tesis para obtener el grado de maestro en Administración y Dirección de Empresas, Costa Rica, Universidad de Costa Rica, 2009, p. 19.

4 "Aumentó en 200\% el número de teletrabajadores entre 2012 y 2016 en Colombia", El País. [Consulta: 16 de mayo, 2017]. Disponible en: http://www.elpais.com.co/tecnologia/aumento-en-200-el-numero-de-teletrabajadores-entre-2012-y-2016-en-colombia.html
} 
por equipos tecnológicos e informáticos en una plataforma comunicacional, reporta sus labores en tiempo real a sus supervisores inmediatos". ${ }^{5}$

Para Sanguineti Raymond "el concepto de teletrabajo se vincula exclusivamente con quienes, de forma originaria o como fruto de un acuerdo de externalización de funciones previamente desarrolladas de forma presencial, desarrollan un trabajo en beneficio de uno o más empresarios, bien desde su domicilio o bien desde otro lugar distinto del habitual u ordinario, sirviéndose de medios o equipos informáticos y de telecomunicaciones para su ejecución y eventualmente para entrar en conexión con el destinatario de su actividad". ${ }^{6}$

En opinión de César Puntriano, el teletrabajo es "un fenómeno caracterizado por que el teletrabajador presta sus servicios a distancia del centro de trabajo tradicional, lo cual supone un cambio de la organización de la unidad empresarial, utilizando de manera esencial medios telemáticos sin los cuales no nos encontraríamos ante el teletrabajo". ${ }^{7}$

Por otro lado, Carmen de la Cámara, en la definición de teletrabajo, destaca dos elementos: trabajo realizado fuera del centro físico de trabajo habitual y el uso de tecnologías informáticas y de comunicación. ${ }^{8}$

Así también, en el diccionario de la lengua española de la RAE, el teletrabajo significa "Trabajo que se realiza desde un lugar fuera de la empresa utilizando las redes de telecomunicación para cumplir con las cargas laborales asignadas”.

Encontramos, asimismo, que en el entorno normativo de la Organización Internacional del Trabajo (огт), el término "trabajo a distancia" no cuenta con una definición propia y remite al término "teletrabajo”. Éste se define como

\footnotetext{
${ }^{5}$ Reyna Támara, Fernando, "El Teletrabajo en las empresas del sistema financiero", Jurídica, Suplemento de análisis legal de El Peruano, núm. 601, p. 5.

${ }^{6}$ Sanguineti Raymond, Wilfredo, "Informe Teletrabajo y Globalización: en busca de Respuestas al desafío de la transnacionalización del empleo", El blog de Wilfredo Sanguineti. [Consulta: 30 de abril, 2017]. Disponible en ttps:// wilfredosanguineti.files.wordpress.com/2009/08/teletrabajo-y-globalizacion-final-informe.pdf

7 PUnTRIANo, CÉsAR, "El teletrabajo, nociones básicas y breve aproximación al ejercicio de los derechos colectivos de los teletrabajadores", lus et veritas, año 14, núm. 29, p. 157.

${ }^{8}$ De la Cámara, Carmen, en su artículo El Teletrabajo. Un indicador de Cambio en el Mercado de Trabajo, señala: "Hay dos elementos esenciales a tener en cuenta para explicar qué se entiende por teletrabajo. El primero se desprende de la interpretación etimológica de la palabra: el prefijo tele -que proviene del término griego "telos"(a distancia)_ indica que el teletrabajo es trabajo realizado lejos del centro físico de trabajo habitual, donde se ubica el empleador. El segundo elemento esencial lo constituye el hecho de que por teletrabajo no se entiende sólo trabajo a distancia, sino que engloba en su significación un uso intensivo de las tecnologías informáticas y de comunicación. Fuera de estos dos elementos, no existe un acuerdo general que permita una definición de teletrabajo comprensiva de las diferentes formas que adopta". Martinez Berndt, Ana Maria, Espinoza Céspedes, José Francisco y Patrón Bedoya, Pedro, "Aproximaciones normativas al teletrabajo en el Perú a inicios del siglo xxi", Revista Decir, año 3, núm. 1, p. 194.
} 
trabajo a distancia (incluido el trabajo a domicilio) efectuado con auxilio de medios de telecomunicación o de una computadora. ${ }^{9}$

Encontramos otra definición de teletrabajo en el Acuerdo Marco Europeo. Así, se señala que: "El teletrabajo es una forma de organización y/o de realización del trabajo, utilizando las tecnologías de la información en el marco de un con trato o de una relación de trabajo, en la cual un trabajo que podría ser realizado igualmente en los locales de la empresa se efectúa fuera de estos locales de forma regular. El presente acuerdo cubre a los teletrabajadores. Se entiende por teletrabajador toda persona que efectúa teletrabajo según la definición anterior". ${ }^{10}$

Debido a que el teletrabajo comprende diversas situaciones y prácticas cambiantes, en el acuerdo mencionado se consideró una definición de teletrabajo que haga posible considerar las diversas formas de teletrabajo regular y reconocer la importancia del teletrabajo en la modernización de la organización del trabajo. Asimismo, se deben reconocer sus beneficios para las empresas, organizaciones y teletrabajadores, especialmente aquellos que adolecen de discapacidad promoviendo con ello la inclusión laboral. ${ }^{11}$

En el ordenamiento jurídico peruano, en la Ley 30036 que regula el teletrabajo, los aspectos más resaltantes al definir el teletrabajo son: el uso de las TIc; el trabajo subordinado de labores y la no presencia física del teletrabajador. Así, el artículo 2 de la norma acotada señala lo siguiente.

Artículo 2. Definición de teletrabajo

El teletrabajo se caracteriza por el desempeño subordinado de labores sin la presencia física del trabajador, denominado "teletrabajador", en la empresa con la que mantiene vínculo laboral, a través de medios informáticos, de telecomunicaciones y análogos, mediante los cuales se ejercen a su vez el control y la supervisión de las labores.

Son elementos que coadyuvan a tipificar el carácter subordinado de esta modalidad de trabajo la provisión por el empleador de los medios

\footnotetext{
9 Ushakova, TATSIANA, "El Derecho de la oit para el trabajo a distancia: ¿una regulación superada o todavía aplicable?", Revista Internacional y Comparada, p. 2. Disponible en: http://www.europeanrights.eu/public/commenti/Bronzini21-USHAAKOVA-332-686-1-PB.pdf

10 "Acuerdo Marco Europeo sobre Teletrabajo de fecha 16 de julio de 2002", ccoo Federación Servicios. [Consulta: 04 de mayo, 2017]. Disponible en: https://www.ccoo-servicios.es/archivos/bbva/20110727_acuerdo_marco_europeo_teletrabajo.pdf

${ }^{11}$ Cfr. "Acuerdo Marco Europeo sobre Teletrabajo de fecha 16 de julio de 2002", ccoo Federación Servicios. [Consulta: 04 de mayo, 2017]. Disponible en: https://www.ccoo-servicios.es/archivos/bbva/20110727_acuerdo_marco_europeo_teletrabajo.pdf
} 
físicos y métodos informáticos, la dependencia tecnológica y la propiedad de los resultados, entre otros.

$\mathrm{Al}$ respecto, cabe señalar que es un tipo de teletrabajo el que regula la Ley 30036 ya que existen otros tipos, de acuerdo con las necesidades de las empresas y organizaciones. Carmen de la Cámara, para clasificar los tipos de teletrabajo, hace uso del criterio de la localización de la prestación. ${ }^{12}$

\section{Características del teletrabajo}

El teletrabajo tiene como características resaltantes el empleo de medios físicos e informáticos, además de la subordinación de labores sin la presencia física del trabajador.

Sobre este aspecto, Francisco Ortiz Chaparro afirma que "La actividad se desarrolla para una empresa o institución (un empleador) sin acudir al centro de trabajo tradicional. A la empresa. A «la oficina». En este planteamiento no hacemos cuestión del lugar donde se realice ese trabajo y que no sea el

\footnotetext{
12 De la Cámara, al desarrollar los tipos de teletrabajo, utiliza la clasificación que sigue el criterio de localización de la prestación. Menciona que es posible la combinación de dos o más de ellas. Así, los tipos de teletrabajo que desarrolla son:

"1) El teletrabajo realizado en casa durante toda la jornada laboral y por cuenta ajena. [...]

2) El teletrabajo que se comparte, de manera regular, entre la casa y la oficina. Corresponde al concepto de telecommuting. Dentro de este grupo estarian los trabajadores que corresponden a la descripción idealizada de la era de la información (de sexo masculino, altamente cualificados, bien pagados, cuyas relaciones con la empresa están basadas en la confianza y que tienen autonomía para decidir dónde y cuándo trabajar). [...]

3) El trabajo en un telecentro, también llamado centro de recursos compartidos. Este es un centro dotado de recursos informáticos y telemáticos que se comparte entre varios teletrabajadores, los cuales no forman un grupo de trabajo, sino que desempeñan su trabajo de forma independiente, pero que de esta manera pueden disponer entre todos ellos de una infraestructura que, de otro modo, no resultaría asequible o quedaría infrautilizada [...]

4) El teletrabajo en una sede remota, a la que se llama habitualmente "oficina satélite". Esta es una unidad separada conectada electrónicamente con la sede principal, donde se realizan algunas actividades que pueden ser desarrolladas de forma independiente. Otra modalidad de sede remota es el denominado "sistema distribuido" según el cual distintas unidades descentralizadas, ubicadas en distintos lugares geográficos, desarrollan de forma interdependiente las diversas fases de un solo proceso de trabajo, comunicándose entre ellas por vía telemática. [...]

5) El teletrabajo móvil o nómada: no se realiza en un lugar determinado, sino que el trabajador se desplaza frecuentemente y trabaja desde el sitio en que se encuentra en cada momento. Este tipo de trabajo está aumentando. [...]

6) El teletrabajo autónomo. Se identifica como teletrabajo la subcontratación de determinados procesos, si se realizan utilizando las tecnologias de la información y de las comunicaciones". Martinez Berndt, Ana Maria, Espinoza Céspedes, José Francisco y Patrón Bedoya, Pedro, "Aproximaciones normativas al teletrabajo en el Perú a inicios del siglo xxl", Revista Decir, año 3, núm. 1.
} 
tradicional: el domicilio, el telecentro, el propio vehículo en que el teletrabajador se traslada para visitar clientes, etcétera. Simplemente, se realiza fuera de los contornos inmediatos del lugar en que se evalúan sus resultados. Además, el empleador no tiene posibilidad física de observar la ejecución de la prestación por parte del trabajador". ${ }^{13}$

Asimismo, en la Ley 30036 y su reglamento aprobado por el Decreto Supremo 017-2005-TR se resalta como característica de dicha modalidad de trabajo el uso de las тіc. Así el segundo considerando del Reglamento acotada señala lo siguiente.

Que, la Ley No 30036 regula el teletrabajo como una modalidad especial de prestación de servicios caracterizada por la utilización de tecnologías de la información y las telecomunicaciones, en las instituciones públicas y privadas" (las cursivas son nuestras).

En este punto cabe considerar las diferencias entre el teletrabajo y el trabajo a domicilio, que hoy en día ya están claramente definidas. Así, en el ordenamiento jurídico peruano, el teletrabajo y el trabajo a domicilio se encuentran reguladas separadamente.

Así el trabajo a domicilio, de uso más frecuente en actividades manuales, está regulada en el artículo 155 del Decreto Legislativo 728, Ley del Fomento del Empleo:

Artículo 155.- Trabajo a domicilio es el que se ejecuta, habitual o temporalmente, de forma continua o discontinua, por cuenta de uno o más empleadores, en el domicilio del trabajador o en el lugar designado por ésta, sin supervisión directa e inmediata del empleador. El empleador tiene la facultad de establecer las regulaciones de la metodología y técnicas del trabajo a realizarse.

En la producción de bienes inmateriales el derecho a la propiedad intelectual del bien producido lo reserva el empleador, salvo que medie pacto expreso en contrario.

Como podrá apreciarse, en dicha definición no se hace mención expresa al uso de las тाс como la Ley 30036 que regula el teletrabajo.

\footnotetext{
13 Ortiz Chaparro, Francisco, El teletrabajo. Una nueva sociedad laboral en la era de la tecnología, Madrid, McGraw-Hill - Interamericana de España, 1997.
} 
Doctrinariamente se podría indicar que ambas son especies del trabajo a la distancia. El uso de las тाс caracteriza al teletrabajo, mientras que el trabajo a domicilio se basa en actividades manuales. Es la preparación del trabajador lo que diferencia también a dichas modalidades de trabajo.

Para otros como Francisco Verdera el teletrabajo es una modalidad del trabajo a domicilio. Así, señala que el teletrabajo es:

Una modalidad moderna y reciente de trabajo a domicilio es la del trabajo a distancia, también denominado tele-trabajo. El aumento en el uso de la tecnología de la información -avance en el uso de computadoras personales y las telecomunicaciones- permite que el procesamiento de la información pueda ser efectuado y administrado a distancia.

Los trabajadores inmersos en esta modalidad son profesionales y técnicos de alta calificación -o entrenados en el uso de computadorasque trabajan en sus domicilios y que se comunican mediante módem $u$ otros medios con quienes los contratan. Se tratará de trabajo a domicilio en la medida que exista subordinación o relación laboral de dependencia. En cambio, en el caso más frecuente en países desarrollados, del trabajo electrónico a domicilio, se distingue del trabajo a domicilio convencional porque "...se basa en un conjunto completamente distinto de calificaciones profesionales, modos de organización y enlace con la sede del empleador. ${ }^{14}$

Sobre el trabajo a domicilio dicho autor señala, asimismo, que:

El trabajo a domicilio se encuentra bastante concentrado en algunas subramas de la industria (confecciones y textiles, calzado de cuero, carpintería de madera) y con predominio de mujeres en el caso de confecciones. También se concentra en determinadas zonas, como son los distritos de El Porvenir en Trujillo y Villa El Salvador, los alrededores del Jirón Gamarra, y está presente en la producción artesanal. ${ }^{15}$

En nuestra opinión, es la prestación del servicio que se hace en lugar distinto del establecimiento del empleador y la relación laboral existente las características

\footnotetext{
${ }^{14}$ Verdera, Francisco, "Trabajadores a Domicilio en el Perú", International Labour Organization. [Consulta: 28 de abril,

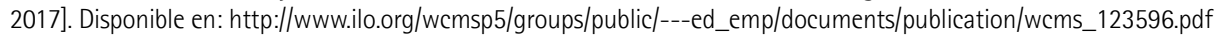
${ }^{15}$ Verdera, Francisco, "Trabajadores a Domicilio en el Perú", International Labour Organization. [Consulta: 28 de abril, 2017]. Disponible en: http://www.ilo.org/wcmsp5/groups/public/---ed_emp/documents/publication/wcms_123596.pdf
} 
comunes de ambas, así como la protección que el Estado les brinda (remuneración, beneficios, entre otros).

\section{Beneficios y calidad de vida}

Los beneficios del teletrabajo se extienden al teletrabajador, empleador y a la sociedad en general. Contribuyen a la mejor calidad de vida de las personas.

Evita conflictos, gastos adicionales y estrés por desplazamiento al centro laboral. Asimismo, quienes cuentan con vehículo ven con preocupación la afectación de su economía por la adquisición de gasolina y otros combustibles que contaminan el medio ambiente. Por el contrario, el teletrabajo contribuye al ahorro de tiempo, dinero, protección de la salud y del medio ambiente.

El teletrabajo contribuye al fortalecimiento de la familia al permitir que el teletrabajador comparta más tiempo ella. Esto dependerá, claro está, de la organización de sus tiempos. El estrechamiento de los lazos familiares contribuye al desarrollo integral de los hijos.

Para la empresa y para las entidades del Estado, el teletrabajo permite ahorrar gastos (infraestructura, agua, luz, refrigerio) y el mejor cumplimiento de sus objetivos y funciones, acorde con los tiempos actuales en el que se promueve y regula sobre el uso de las tic. Se incrementa la productividad de las empresas, las cuales se encuentran satisfechas con los logros en los que el teletrabajador ha sido partícipe.

El Estado permite mejorar la calidad de vida especialmente de las poblaciones vulnerables. Es su deber la promoción, protección e implementación del teletrabajo. El trabajo es un derecho y un deber, base del bienestar social y un medio de realización de la persona, conforme con el artículo 22 de la Constitución Política del Perú de 1993.

Sobre la calidad de vida, María Galván señala:

Sabemos que para una buena calidad de vida tanto material como espiritual, interviene el factor económico, ya que de acuerdo a los ingresos personales se puede reflejar un bienestar social, la cuestión que aquí aparece es que no todos tenemos acceso a tener bienes de diversa índole, pues la mayoría de la población no tiene lo básico en su hogar, y que de acuerdo a datos estadísticos, los individuos sobreviven con alrededor de quince a veintiséis pesos diarios, lo cual nos demuestra que no 
todos tenemos lo elemental para considerar que tenemos una calidad de vida, que comprende aspectos de salud, educación. ${ }^{16}$

Una adecuada calidad de vida coadyuva a la mejora de la persona y de la sociedad, ya que se satisfacen las necesidades y proyectos de vida. La inclusión laboral es por ello un desafío para el Estado peruano que apuesta por el teletrabajo para ayudar a que las personas accedan a un puesto de trabajo.

\section{Aspectos resaltantes de la Ley 30036 y su Reglamento}

Entre los aspectos más importantes de la Ley $30036^{17}$ señalamos los siguientes.

La regulación del teletrabajo se sustenta en los artículos 22 y 23 de la Constitución Política del Perú. Así, el artículo 22 establece que el trabajo es un deber y un derecho, base del bienestar social y un medio de realización de la persona. El Tribunal Constitucional del Perú indica:

el derecho al trabajo supone la adopción por parte del Estado de una política orientada a que la población acceda a un puesto de trabajo; si bien hay que precisar que la satisfacción de este aspecto de este derecho constitucional implica un desarrollo progresivo y según las posibilidades del Estado. El segundo aspecto del derecho es el que resulta relevante para resolver la causa. Se trata del derecho al trabajo entendido como proscripción de ser despedido salvo por causa justa. ${ }^{18}$

La Ley 30036 tiene como objeto, conforme a su artículo 1, "regular el teletrabajo como una modalidad especial de prestación de servicios caracterizada por la utilización de tecnologías de la información y las telecomunicaciones (тіс), en las instituciones públicas y privadas, y promover políticas públicas para garantizar su desarrollo".

En cuanto al desarrollo del teletrabajo, aspecto relevante, entre otros, para la aprobación de la Ley 30036, el estudio efectuado por Technology Landscape

\footnotetext{
${ }^{16}$ Galván, Maria, "¿Qué es la calidad de vida?", Universidad Autónoma del Estado de Hidalgo. [Consulta: 3 de mayo, 2017]. Disponible en https://www.uaeh.edu.mx/scige/boletin/prepa2/n2/m2.html

17 "Exposición de Motivos del Reglamento de la Ley N 30036, Ley que Regula el Teletrabajo", Sistema peruano de información juridica. [Consulta: 30 de abril, 2017]. Disponible en: http://spij.minjus.gob.pe/Graficos/Peru/2015/ Noviembre/03/EXP-DS-009-2015-TR.pdf

${ }_{18}$ Sentencia del Tribunal Constitucional, Lima, Sindicato Unitario de Trabajadores de Telefónica del Perú S. A. y Fetratel, exp. núm. 1124-2001-AA/TC, Fundamento 12.
} 
2020, solicitado por la corporación multinacional Citrix, establece que para el 2020 uno de cada dos trabajadores realizará sus labores desde su casa o en algún otro lugar, pero no en una oficina a la que acuda todos los días con una jornada de trabajo. ${ }^{19}$

La Ley 30036, asimismo, establece en su artículo 3 las reglas en cuanto al uso y cuidado de los equipos que se entregan al teletrabajador. Si es el teletrabajador quien aporta los equipos, se dispone que corresponde al empleador compensar los gastos. Si no tuviera equipos, pero le son proporcionados por terceras personas o hace uso de cabinas de internet, también el empleador debe asumir los gastos. De conformidad con el artículo 11 del Reglamento de la Ley: "Los bienes y servicios brindados por el empleador o entidad pública como condiciones de trabajo no tienen carácter remunerativo para ningún efecto legal."

El carácter voluntario y reversible del teletrabajo se encuentra establecido en el artículo 4 de la Ley, aunque la propia norma señala que el empleador puede no aceptar dicha solicitud. "El empleador puede denegar dicha solicitud en uso de su facultad directriz".

En cuanto a los derechos y obligaciones laborales, el teletrabajador tiene los mismos derechos y obligaciones establecidos para los trabajadores del régimen laboral de la actividad privada y puede utilizarse en todas las modalidades establecidas para dicho régimen. Es obligatorio que el contrato de trabajo sea por escrito, como lo determina el artículo 5 de la Ley. ${ }^{20}$

Cabe resaltar que la Ley, en su segunda disposición complementaria final, estableció un plazo de noventa días hábiles de entrada en vigencia para que el Ministerio de Trabajo y Promoción del Empleo formule las políticas públicas referidas al teletrabajo. Lo anterior tiene la finalidad de garantizar su desarrollo y preferente utilización a favor de las poblaciones vulnerables. Para dicho cometido es necesaria la coordinación con la Autoridad Nacional del Servicio Civil (Servir), con la Oficina Nacional de Gobierno Electrónico e Informática (ongeI), con el Consejo Nacional para la Integración de la Persona con Discapacidad (Conadis) y con la Comisión Multisectorial para el Seguimiento y

\footnotetext{
19 "Exposición de Motivos del Reglamento de la Ley N³0036, Ley que Regula el Teletrabajo", Sistema peruano de información jurídica. [Consulta: 30 de abril, 2017]. Disponible en: http://spij.minjus.gob.pe/Graficos/Peru/2015/ Noviembre/03/EXP-DS-009-2015-TR.pdf

$20 \mathrm{E} /$ régimen laboral de la actividad privada en el Perú se encuentra regulado en el Decreto Legislativo $\mathrm{N}^{\circ} 728$-Ley de Fomento del Empleo, publicado en el Diario Oficial El Peruano el 12 de noviembre de 1991. En cuanto a las modalidades de dicho régimen, el artículo 96 de la norma referida señala que "Los contratos de trabajo sujetos a modalidad pueden celebrarse cuando asi lo requieran las necesidades del mercado o mayor producción de la empresa, así como cuando lo exija la naturaleza temporal o accidental del servicio que se va a prestar o de la obra que se ha de ejecutar, excepto los contratos de trabajo intermitentes o de temporada que por su naturaleza puedan ser permanentes".
} 
Evaluación del Plan de Desarrollo de la Sociedad de la Información en el Perú (Codesi).

$\mathrm{Al}$ respecto, el Ministerio de la Mujer y Poblaciones Vulnerables (MIMP) y el Ministerio de Trabajo y Promoción del Empleo (MTPE) han firmado un convenio interinstitucional para promover el teletrabajo para las personas con discapacidad. Según la Encuesta Nacional Especializada sobre Discapacidad (Enedis), 2012, del Instituto Nacional de Estadística (INEI), en el Perú existe 1575402 personas con algún tipo de discapacidad: 5.2\% de la población total.

Por su parte, la Autoridad Nacional del Servicio Civil (Servir) es la encargada de determinar la cuota para el teletrabajo en el sector público.

En cuanto al Decreto Supremo 017-2015-TR, Reglamento de la Ley de Teletrabajo, las disposiciones legales más resaltantes son las que se indican a continuación.

La Ley dispuso su reglamentación la cual se aprobó por Decreto Supremo 017-2015-TR. El Reglamento cuenta con una exposición de motivos donde se señala lo siguiente:

Considerando las particularidades del sector público, el Reglamento prevé disposiciones comunes, aplicables al sector público y privado, así como disposiciones especiales aplicables únicamente a las entidades públicas. Adicionalmente, se prevén otro tipo de disposiciones, entre las cuales, se establecen reglas que favorecen la aplicación de la modalidad de teletrabajo a determinados sectores de la población (madres de familia, personas discapacitadas, etc.). Asimismo, entre otras disposiciones, el Reglamento desarrolla nuevos supuestos de infracciones laborales relacionadas al teletrabajo. ${ }^{21}$

En cuanto al ámbito de aplicación de la Ley de Teletrabajo, el artículo II del título preliminar del reglamento establece:

Se encuentran comprendidos dentro del ámbito de aplicación de la Ley 215 y el Reglamento aquellos trabajadores y servidores civiles que prestan servicios bajo la modalidad de teletrabajo; así como las personas naturales o jurídicas y entidades públicas que los emplean. La Ley y el Reglamento serán de aplicación a: a) Los trabajadores y servidores

\footnotetext{
21 "Exposición de Motivos del Reglamento de la Ley N³0036, Ley que Regula el Teletrabajo", Sistema peruano de información juridica. [Consulta: 30 de abril, 2017]. Disponible en: http://spij.minjus.gob.pe/Graficos/Peru/2015/ Noviembre/03/EXP-DS-009-2015-TR.pdf
} 
civiles cuyas labores se ejecuten en territorio nacional; b) Los contratos, resoluciones de incorporación o designación y adendas o acuerdos, por los que se establezca la modalidad de teletrabajo, o el cambio de modalidad presencial por la de teletrabajo y viceversa; suscritos o emitidos en el país.

La Ley y el Reglamento se aplica al teletrabajador en las siguientes situaciones: que preste servicios en el país independientemente del lugar donde se haya celebrado el contrato de trabajo; que siendo contratado en el país preste servicios en el extranjero bajo la modalidad de teletrabajo. ${ }^{22}$

El reglamento establece definiciones como el teletrabajo, teletrabajador, titular de la entidad, puesto, entidad pública, compensación, entre otros. Esto evitará errores de interpretación cuando se aplique la Ley de Teletrabajo y su Reglamento.

También regula principios como la voluntariedad, reversibilidad, igualdad de trato, conciliación entre la vida personal, familiar y laboral. Estos coadyuvan en la mejor aplicación del teletrabajo, particularmente cuando surgen problemas de interpretación. Dichos principios, como se señala expresamente en su artículo iv, orientan la aplicación de dicha modalidad que promueve una forma de empleo no convencional.

Un tema que merece especial mención es el pago de la compensación. Deberá efectuarlo el empleador en caso de que los medios informáticos, de telecomunicaciones y análogos sean proporcionados por el teletrabajador. $\mathrm{Al}$ respecto, en el caso del sector público dicho pago estará sujeto a las disposiciones de carácter presupuestal aplicables a las entidades del sector. En caso del sector privado, se realiza el pago en dinero y en moneda de curso legal.

En cuanto a las formas de teletrabajo, que no constituye un régimen laboral especial, el reglamento establece dos formas. La forma completa significa que el teletrabajador presta sus servicios fuera del centro de trabajo o del local de la entidad pública. En la forma mixta, el teletrabajador presta servicios de forma alternada dentro y fuera del centro de trabajo o local de la entidad pública. La ocasionalidad fuera del centro de trabajo o local de la entidad pública no califica al trabajador como teletrabajador, de acuerdo con el artículo 2 del Reglamento de la Ley.

\footnotetext{
${ }^{22}$ Cfr. "Exposición de Motivos del Reglamento de la Ley N³0036, Ley que Regula el Teletrabajo", Sistema peruano de información jurídica. [Consulta: 30 de abril, 2017]. Disponible en: http://spij.minjus.gob.pe/Graficos/Peru/2015/ Noviembre/03/EXP-DS-009-2015-TR.pdf
} 
En cuanto a la jornada de trabajo o de servicio, se busca promover con su regulación que el teletrabajador se organice adecuadamente para prestar sus servicios y atender a su familia y que la empresa o entidad pública obtenga los resultados esperados. Por ello, se regula en el artículo 3 del reglamento que "Las exclusiones a la jornada máxima de trabajo o de servicio previstas en las normas que regulan la jornada de trabajo en los sectores público y privado se aplican al teletrabajo". Se establece además que los teletrabajadores pueden prestar servicios en jornadas a tiempo parcial o en sistemas de media jornada sólo si éstos se encuentran sujetos a fiscalización inmediata de la jornada.

En el artículo 4 del reglamento se regula la variación de la modalidad convencional de la prestación de servicios a la de teletrabajo y su reversión a la modalidad convencional. La primera es voluntaria; la segunda debe cumplir con reglas expresas. Si son cuestionadas ambas situaciones por el trabajador, servidor civil o teletrabajador, se deberá observar las normas al régimen al que pertenezcan.

En cuanto a los derechos y beneficios del teletrabajador detallados en los artículos 6 y 7 del Reglamento, se establece la capacitación sobre los medios informáticos, de telecomunicaciones y análogos, datos personales, propiedad intelectual, seguridad de la información; intimidad, privacidad e inviolabilidad de las comunicaciones y documentos privados del teletrabajador; protección de la maternidad y el periodo de lactancia de la teletrabajadora; seguridad y salud en el trabajo y libertad sindical. Como obligaciones del teletrabajador, se señala que deberá cumplir con la normativa vigente sobre seguridad de la información, protección y confidencialidad de los datos y seguridad y salud en el trabajo; disponibilidad para las coordinaciones con el empleador o entidad pública, en caso de ser necesario; guardar confidencialidad de la información; y limitarse al uso personal de los elementos y medios para la realización de las labores entregados por el empleador o entidad pública.

El artículo 8 del reglamento se refiere expresamente a las poblaciones vulnerables. El empleador o entidad pública debe dar preferencia a las poblaciones vulnerables para prestar servicios bajo la modalidad de teletrabajo y deberán evaluar su aplicación para garantizar el cumplimiento de la cuota de empleo de las personas con discapacidad, de conformidad con la Ley 29973. Además, se debe garantizar la continuidad de la prestación de servicio de mujeres gestantes y durante el periodo de lactancia, trabajadores y servidoras civiles responsables del cuidado de niños, adultos mayores, personas con discapacidad o familiares directos que se encuentren con enfermedad en estado grave o terminal o sufran accidente grave. 
Para la imposición de sanciones en materia de teletrabajo, la única disposición complementaria modificatoria del reglamento modifica el artículo 24 del Reglamento de la Ley General de Inspección del Trabajo, para incorporar como infracciones las siguientes conductas:

24.16. Aplicar el cambio de modalidad de un trabajador convencional a la modalidad de teletrabajo o viceversa sin su consentimiento.

24.17 Aplicar la reversión sin cumplir con los requisitos establecidos por ley.

24.18 No cumplir con las obligaciones referidas a la capacitación del teletrabajador previstas en las normas de la materia.

24.19 No cumplir con el pago de la compensación por las condiciones de trabajo asumidas por el teletrabajador.

\section{Políticas públicas y el teletrabajo}

El derecho al trabajo es un derecho humano que todo Estado debe promover y garantizar. Así, el artículo 22 de la Constitución Política del Perú establece lo siguiente: "Artículo 22.- El trabajo es un deber y un derecho. Es base del bienestar social y un medio de realización de la persona”. Sobre el trabajo, también el artículo 23 de la Constitución Política señala:

El trabajo, en sus diversas modalidades, es objeto de atención prioritaria del Estado, el cual protege especialmente a la madre, al menor de edad y al impedido que trabajan.

El Estado promueve condiciones para el progreso social y económico, en especial mediante políticas de fomento del empleo productivo y de educación para el trabajo.

Ninguna relación laboral puede limitar el ejercicio de los derechos constitucionales, ni desconocer o rebajar la dignidad del trabajador. Nadie está obligado a prestar trabajo sin retribución o sin su libre consentimiento.

Consciente de su deber al trabajo y de la necesidad de promover las condiciones económicas y sociales que permitan la generación de puestos de trabajo, el Estado peruano emitió la Ley del Teletrabajo. Con ello, sustentó la necesidad de su regulación en las ventajas del teletrabajo para los trabajadores y para 
el medio ambiente, así como para la productividad de las empresas. A ello se suma su flexibilidad y las experiencias comparadas de otros países. ${ }^{23}$

Otro aspecto que se tomó en cuenta para la dación de la acotada norma es el uso cada vez mayor del teletrabajo en las relaciones laborales. Esto constituye una experiencia beneficiosa para brindar trabajo, en especial a las poblaciones vulnerables a las que nos hemos referido, las cuales no pueden desplazarse para trabajar en puestos de empleo convencionales.

Así también el Estado peruano, al regular el teletrabajo por sus beneficios, también ha prescrito expresamente que se debe garantizar su desarrollo promoviendo políticas públicas. En atención a ello y conforme con lo establecido en la segunda disposición complementaria final de la Ley de teletrabajo, mediante Resolución Suprema 422-2013-PCM, se constituyó una Comisión Multisectorial adscrita al Sector Trabajo y Promoción del Empleo para elaborar el informe técnico que contenga propuestas de políticas públicas referidas al teletrabajo.

Cabe resaltar que, luego de culminado el trabajo de la Comisión, mediante la Resolución Ministerial 121-2015-TR, se prepublicó el referido informe para recabar aportes del público.

Entre las medidas de políticas públicas ${ }^{24}$ que propone el referido informe, encontramos las vinculadas a la promoción del teletrabajo en el sector público y privado; su empleo para las poblaciones consideradas vulnerables por presentar mayores dificultades y restricciones para acceder y sostenerse en el mercado de trabajo; y el fortalecimiento de la institucionalidad y el liderazgo vinculado con el teletrabajo. ${ }^{25}$

Asimismo, el Ministerio de Trabajo y Promoción del Empleo, preocupado por la inclusión de las mujeres en este mercado, encuentra en el teletrabajo un tema de interés nacional, un aliado para reducir las brechas entre hombres y mujeres y lograr la igualdad laboral de la mujer.

Entre las actividades desarrolladas y a desarrollar por el Ministerio de Trabajo y Promoción del Empleo para cumplir con su compromiso de lograr un mercado laboral justo, equitativo, solidario e inclusivo, tenemos las siguientes.

\footnotetext{
${ }^{23}$ Como Colombia, España, Argentina, Costa Rica, Chile y EEUU.

${ }^{24}$ Las que deben formularse con enfoque en los derechos humanos a fin que se atiendan las necesidades fundamentales de los ciudadanos quienes tienen derecho a una vida digna.

${ }^{25}$ Cfr. "Exposición de Motivos del Reglamento de la Ley N 30036, Ley que Regula el Teletrabajo", Sistema peruano de información juridica. [Consulta: 30 de abril, 2017]. Disponible en: http://spij.minjus.gob.pe/Graficos/Peru/2015/ Noviembre/03/EXP-DS-009-2015-TR.pdf
} 
En noviembre del año 2016, el Ministerio de Trabajo y Promoción del Empleo firmó un pacto nacional con los gobiernos regionales, gremios empresariales, empresas y la sociedad civil. Los compromisos que se asumieron fueron los siguientes:

- Sector público: desarrollar actividades de promoción y difusión de políticas, programas y estrategias relacionadas con el fomento del teletrabajo.

- Gobiernos regionales: la implementación progresiva de servicios de información y orientación sobre teletrabajo en los Centro de Empleo del MTPE, y la suscripción de pactos regionales.

- Gremios empresariales: impulsarán una red de empresas para implementar el teletrabajo; las universidades, centros de investigación, organismos internacionales y sociedad civil desarrollarán estudios sobre los beneficios y el impacto de esta modalidad en el Perú. ${ }^{26}$

Son más de 648 los teletrabajadores en la actualidad, de los cuales 145 laboran en 145 empresas de diferentes rubros del país. Las labores más requeridas son asesor de negocios, productos farmacéuticos, empleados de servicios financieros, técnicos de negocios, productos financieros, empleados de servicios financieros, técnicos en servicios informáticos, telefonistas y contadores. ${ }^{27}$

Se han inaugurado dos servicios descentralizados de información y orientación sobre Teletrabajo en la región Lambayeque y en la región Arequipa. ${ }^{28}$ Se prevé implementar dichos servicios también en Tacna, La Libertad, Piura y Callao. $^{29}$

Entre las empresas peruanas que emplean el teletrabajo tenemos: MDS Solutions, KPMG, BCP, Neptunia, Edelnor, Interbank, Choice Ari Courier del Perú, IBM, Cisco Systems, Repsol, Sociedad Agrícola Virú. ${ }^{30}$

\footnotetext{
${ }^{26}$ Cfr. "Gobierno firma pacto para promover el teletrabajo en el sector público y privado", Teletrabajo. Disponible en: http://teletrabajo.gob.pe/gobierno-firma-pacto-para-promover-el-teletrabajo-en-el-sector-publico-y-privado/ 27 "Gobierno firma pacto para promover el teletrabajo en el sector público y privado", Teletrabajo. Disponible en: http://teletrabajo.gob.pe/gobierno-firma-pacto-para-promover-el-teletrabajo-en-el-sector-publico-y-privado/ 28 "Gobierno firma pacto para promover el teletrabajo en el sector público y privado", Teletrabajo. Disponible en: http://teletrabajo.gob.pe/gobierno-firma-pacto-para-promover-el-teletrabajo-en-el-sector-publico-y-privado/ 29 "Gobierno firma pacto para promover el teletrabajo en el sector público y privado", Teletrabajo. Disponible en: http://teletrabajo.gob.pe/gobierno-firma-pacto-para-promover-el-teletrabajo-en-el-sector-publico-y-privado/ 30 "Experiencias nacionales", Teletrabajo. Disponible en: http://teletrabajo.gob.pe/experiencias-nacionales/
} 


\section{Teletrabajo y gobierno electrónico}

Dado que el teletrabajo hace uso de las TIc, su vinculación con el gobierno electrónico es una realidad. Previamente al desarrollo de este tema, definiremos gobierno electrónico.

Para la Carta Iberoamericana de Gobierno Electrónico, se entienden:

las expresiones de 'Gobierno Electrónico' y de 'Administración Electrónica' como sinónimas, ambas consideradas como el uso de las TIC en los órganos de la Administración para mejorar la información y los servicios ofrecidos a los ciudadanos, orientar la eficacia y eficiencia de la gestión pública e incrementar sustantivamente la transparencia del sector público y la participación de los ciudadanos. Todo ello, sin perjuicio de las denominaciones establecidas en las legislaciones nacionales. ${ }^{31}$

El gobierno electrónico es una innovación continua de los servicios, la participación de los ciudadanos y la forma de gobernar mediante la transformación de las relaciones externas e internas a través de la tecnología, el Internet y los nuevos medios de comunicación. ${ }^{32}$

Para la OCDE, "El Gobierno Electrónico es el uso de las tecnologías de la información y comunicación (TIC), particularmente la Internet, como una herramienta para alcanzar un mejor gobierno". ${ }^{33}$

A su vez, el Banco Mundial señala que "El gobierno electrónico se refiere al uso de tecnologías de información por parte de las agencias gubernamentales que tienen la habilidad de transformar las relaciones entre los ciudadanos, los negocios y otros brazos del gobierno". ${ }^{34}$

Los beneficios que brinda el gobierno electrónico son diversos, particularmente para el Estado. En el plano de políticas públicas, por ejemplo, en el

31 "Carta Iberoamericana de Gobierno Electrónico aprobada por la IX Conferencia Iberoamericana de Ministros de Administración Pública y Reforma del Estado Pucón, Chile, 31 de mayo y 1 de junio de 2007 Adoptada por la XVII Cumbre Iberoamericana de Jefes de Estado y de Gobierno Santiago de Chile, 10 de noviembre de 2007 (Resolución No. 18 de la Declaración de Santiago)". [Consulta: 2 de mayo, 2017]. Disponible en: http://old.clad.org/documentos/ declaraciones/cartagobelec.pdf

32 "Gobierno Electrónico y Gestión Pública", Comisión Económica para América Latina y el Caribe (Cepal). [Consulta: 2 de mayo, 2017]. Disponible en: http://www.cepal.org/ilpes/noticias/paginas/5/39255/gobierno_electronico_anaser.pdf 33 "Gobierno Electrónico y Gestión Pública", Comisión Económica para América Latina y el Caribe (CEPAL). [Consulta: 2 de mayo, 2017]. Disponible en: http://www.cepal.org/ilpes/noticias/paginas/5/39255/gobierno_electronico_anaser.pdf 34 "Gobierno Electrónico y Gestión Pública", Comisión Económica para América Latina y el Caribe (CEPAL). [Consulta: 2 de mayo, 2017]. Disponible en: http://www.cepal.org/ilpes/noticias/paginas/5/39255/gobierno_electronico_anaser.pdf 
campo laboral, los ciudadanos tienen la posibilidad de acceder a un trabajo sin necesidad de desplazarse a determinada empresa. Lo anterior dado que las Tic brindan dicha posibilidad. Otro beneficiado es el propio Estado, el cual coadyuva mediante la implementación del teletrabajo a mejorar la calidad de vida de los ciudadanos.

Asimismo, el Gobierno peruano, al aprobar la política pública de gobierno electrónico (transversal a todas las demás políticas públicas), ha establecido que los funcionarios y servidores públicos deben valerse de los medios electrónicos para el cumplimiento de sus obligaciones y que se debe promover la cultura digital.

Así las cosas, dado que el teletrabajo se basa en el uso de las Tic, y que su aplicación está permitido también en el sector público, corresponde a las entidades del Estado revisar sus funciones. Lo anterior con el fin de establecer los puestos que puedan desempeñarse mediante teletrabajo. Ello está determinado en el artículo 13 del Reglamento de la Ley de Teletrabajo:

Aprobación de la modalidad de teletrabajo en las entidades públicas Las entidades públicas identificarán progresivamente aquellos puestos que puedan desempeñarse a través de la modalidad de teletrabajo, en el marco de sus instrumentos de gestión aprobados.

Asimismo, el titular de la entidad aprobará el informe que establezca, en razón de las características del puesto, cuáles de ellos pueden desempeñarse a través de la modalidad de teletrabajo.

Para ello, se constituirá una Comisión de Teletrabajo, en la cual participen un representante de la Oficina de Recursos Humanos, de la Oficina General de Administración, de la Oficina de Tecnología de la Información y del Titular de la entidad.

La Comisión de Teletrabajo tiene como función elaborar una propuesta de puestos identificados en la entidad pública que pueden desempeñarse a través de la modalidad de teletrabajo.

Asimismo, las entidades del sector público deben observar la cuota mínima de teletrabajo la que debe establecer Servir, de acuerdo con lo establecido en la cuarta disposición complementaria final del reglamento señalado.

El Reglamento también señala la posibilidad de que la entidad pública contrate personal para desempeñarse como teletrabajador cuando se presentan situaciones excepcionales. Al respecto, el artículo 15 señala: 
Las entidades públicas pueden requerir la contratación de servidores civiles que se desempeñen como teletrabajadores desde un inicio de la prestación de servicios, bajo los siguientes supuestos:

a) En circunstancias de caso fortuito o fuerza mayor que requieran que algunas actividades se realicen bajo la modalidad de teletrabajo.

b) Cuando se afecte la prestación de servicios de manera imprevista, y para garantizar su continuidad se puedan realizar algunas actividades bajo la modalidad de teletrabajo.

c) Cuando se produce el incremento extraordinario y temporal de actividades en una determinada entidad.

Esta participación del sector público para promover el teletrabajo requiere de la capacitación obligatoria de sus colaboradores en el uso de las тic. Por ello, también en el Reglamento de la Ley 30036 se ha considerado necesario regular la capacitación de los servidores públicos. Así, la acotada norma regula lo siguiente:

Las entidades públicas deben considerar en la planificación de las necesidades de capacitación de sus servidores civiles, actividades vinculadas a los siguientes temas:

a) Competencias para que los servidores civiles se puedan desempeñar en la modalidad de teletrabajo.

b) Lineamientos y políticas generales de la organización para el teletrabajo.

c) Prevención en salud y riesgos laborales.

d) Uso y manejo de las herramientas de tecnología de la información y la comunicación.

El desarrollo de habilidades y potencialidades de las personas es necesaria para desempeñarse por medios electrónicos. El desarrollo de las Tic obliga a las per223 sonas a estar preparadas para adaptarse a nuevas modalidades de trabajo en el campo laboral, donde están presentes también las tecnologías de la información y las comunicaciones.

$\mathrm{Al}$ respecto, Begoña Eguía e Ixone Alonso señalan:

El nuevo contexto de la Sociedad de la Información y el desarrollo de las Tecnologías de la Información y la Comunicación inciden en los 
ámbitos económico, político, social y cultural. La transformación del mercado laboral constituye un exponente claro de los cambios propiciados por la denominada economía digital. Por un lado, emergen nuevos empleos y perfiles profesionales que requieren el dominio de estas tecnologías. Por otro lado, surge un nuevo modelo de búsqueda de empleo: la Red actúa de elemento intermediario entre oferentes y demandantes". 35

Sobre las ventajas del teletrabajo dichos autores opinan lo siguiente.

Las transformaciones tecnológicas están contribuyendo, asimismo, a la creación de una nueva modalidad laboral: el teletrabajo (es decir, el trabajo a distancia utilizando medios telemáticos). Una de las ventajas que presenta esta modalidad es la mayor flexibilidad en la organización del trabajo ya que el uso de las TIc permite superar las barreras espaciales y temporales. Los horarios y la ubicación geográfica del cliente o proveedor no constituyen limitación alguna para establecer las relaciones económicas. Los esfuerzos realizados para impulsar esta nueva fórmula de empleo ayudarán, por tanto, a mejorar el acceso al mercado laboral de colectivos con mayores dificultades de inserción: mujeres con cargas familiares, personas con discapacidades, individuos de mayor edad".

\section{Conclusiones}

Del análisis efectuado sobre el teletrabajo concluimos, en primer lugar, que el teletrabajo se caracteriza por hacer uso de la tecnología de las comunicaciones (тіс). A diferencia del trabajo a domicilio, el esfuerzo que realiza el teletrabajador se debe exclusivamente al uso de las Tic.

En segundo lugar, el teletrabajo se caracteriza por su carácter subordinado. Los elementos que coadyuvan a tipificar dicho carácter son la provisión por el empleador de los medios físicos y métodos informáticos, la dependencia tecnológica y la propiedad de los resultados, entre otros elementos.

\footnotetext{
${ }^{35}$ Begoña, Eguia e IXone, Alonso, "El desarrollo de las tecnologías de la información y la comunicación: un nuevo reto para el mercado de trabajo", Scripta Nova Revista electrónica de Geografía y Ciencias Sociales, vol. 6, núm. 119. [Consulta: 04 de mayo, 2017]. Disponible en http://www.ub.edu/geocrit/sn/sn119-74.htm
} 
En tercero, los ciudadanos, particularmente las poblaciones vulnerables, tienen la posibilidad de acceder a un trabajo sin necesidad de desplazarse a su centro laboral. Con ello consiguen ingresos para mejorar su calidad de vida. Para ello, es necesario que estén preparados en el uso de las Tic.

En cuarto lugar, el teletrabajo es una modalidad de trabajo que al ser aprobado e impulsado por el Estado contribuye a la inclusión laboral y con ello se protege y garantiza el derecho al trabajo que es un derecho humano, base del bienestar social y un medio de realización de la persona.

En quinto lugar, los beneficios que brinda el gobierno electrónico son diversos, particularmente para el Estado. Las políticas públicas laborales deben elaborarse con enfoque en derechos humanos, como se viene adoptando en el Perú. Los ciudadanos, con ello, tienen la posibilidad de acceder a un puesto de trabajo. Por otro lado, se protege el medio ambiente.

En sexto lugar, la tendencia es el aumento en el uso del teletrabajo. El sector público y el sector privado deben adecuar sus funciones de tal manera que se permita la aplicación del teletrabajo.

\section{Bibliografía}

“Acuerdo Marco Europeo sobre Teletrabajo de fecha 16 de julio de 2002", ccoo Federación Servicios. [Consulta: 04 de mayo, 2017]. Disponible en: https://www. ccoo-servicios.es/archivos/bbva/20110727_acuerdo_marco_europeo_teletrabajo.pdf

"Aumentó en 200\% el número de teletrabajadores entre 2012 y 2016 en Colombia", El País. [Consulta: 16 de mayo, 2017]. Disponible en: http://www.elpais. com.co/tecnologia/aumento-en-200-el-numero-de-teletrabajadores-entre-2012-y-2016-en-colombia.html

Begoña, Eguía e Ixone, Alonso, "El desarrollo de las tecnologías de la información y la comunicación: un nuevo reto para el mercado de trabajo", Scripta Nova Revista electrónica de Geografía y Ciencias Sociales, vol. 6, núm. 119. [Consulta: 04 de mayo, 2017]. Disponible en http://www.ub.edu/geocrit/sn/ sn119-74.htm

"Carta Iberoamericana de Gobierno Electrónico aprobada por la IX Conferencia Iberoamericana de Ministros de Administración Pública y Reforma del Estado Pucón, Chile, 31 de mayo y 1 de junio de 2007 Adoptada por la XVII Cumbre Iberoamericana de Jefes de Estado y de Gobierno Santiago de Chile, 10 de noviembre de 2007 (Resolución No. 18 de la Declaración de Santiago)”. [Consulta: 2 de mayo, 2017]. Disponible en: http://old.clad.org/documentos/ declaraciones/cartagobelec.pdf 
CAstro, Luis, Propuesta para un modelo de teletrabajo en la Universidad de Costa Rica, tesis para obtener el grado de maestro en Administración y Dirección de Empresas, Costa Rica, Universidad de Costa Rica, 2009.

“Exposición de Motivos del Reglamento de la Ley $\mathrm{N}^{\circ}$ 30036, Ley que Regula el Teletrabajo", Sistema peruano de información jurídica. [Consulta: 30 de abril, 2017]. Disponible en: http://spij.minjus.gob.pe/Graficos/Peru/2015/Noviembre/03/EXP-DS-009-2015-TR.pdf

Galván, MaríA, “¿Qué es la calidad de vida?”, Universidad Autónoma del Estado de Hidalgo. [Consulta: 3 de mayo, 2017]. Disponible en https://www.uaeh.edu. $\mathrm{mx} / \mathrm{scige} /$ boletin/prepa2/n2/m2.html

“Gobierno Electrónico y Gestión Pública”, Comisión Económica para América Latina $y$ el Caribe (CEPAL). [Consulta: 2 de mayo, 2017]. Disponible en: http://www. cepal.org/ilpes/noticias/paginas/5/39255/gobierno_electronico_anaser.pdf

“Gobierno firma pacto para promover el teletrabajo en el sector público y privado", Teletrabajo. Disponible en: http://teletrabajo.gob.pe/gobierno-firma-pacto-para-promover-el-teletrabajo-en-el-sector-publico-y-privado/

Martínez Berndt, Ana María, Espinoza Céspedes, José Francisco y Patrón Bedoya, Pedro, "Aproximaciones normativas al teletrabajo en el Perú a inicios del siglo xxI", Revista Decir, año 3, núm. 1.

Ortiz Chaparro, Francisco, El teletrabajo. Una nueva sociedad laboral en la era de la tecnologia, Madrid, McGraw-Hill - Interamericana de España, 1997.

PUNTRIANO, CÉSAR, "El teletrabajo, nociones básicas y breve aproximación al ejercicio de los derechos colectivos de los teletrabajadores”, Ius et veritas, año 14, núm. 29.

Reyna TÁmARA, Fernando, "El Teletrabajo en las empresas del sistema financiero”, Jurídica, Suplemento de análisis legal de El Peruano, núm. 601.

Sentencia del Tribunal Constitucional, Lima, Sindicato Unitario de Trabajadores de Telefónica del Perú S. A. y Fetratel, exp. núm. 1124-2001-AA/TC, Fundamento 12. SAnguineti Raymond, Wilfredo, "Informe Teletrabajo y Globalización: en busca de Respuestas al desafío de la transnacionalización del empleo”, El blog de Wilfredo Sanguineti. [Consulta: 30 de abril, 2017]. Disponible en ttps://wilfredosanguineti.files.wordpress.com/2009/08/teletrabajo-y-globalizacion-final-informe.pdf

Ushakova, TATsiana, "El Derecho de la oit para el trabajo a distancia: ¿una regulación superada o todavía aplicable?”, Revista Internacional y Comparada. Disponible en: http://www.europeanrights.eu/public/commenti/Bronzini21-USHAAKOVA-332-686-1-PB.pdf

VARGAS RAschio, Tino, La respuesta del ordenamiento laboral peruano frente al teletrabajo, Tesis para obtener el título profesional de abogado, San Miguel, 2010.

Verdera, Francisco, “Trabajadores a Domicilio en el Perú”, International Labour Organization. [Consulta: 28 de abril, 2017]. Disponible en: http://www.ilo.org/wcmsp5/groups/public/---ed_emp/documents/publication/wcms_123596.pdf 